\title{
POLÍTICAS PÚBLICAS PARA CONTRARRESTAR LA ESCALADA DE LAS ENFERMEDADES CRÓNICO NO TRANSMISIBLES EN MÉXICO (2001-2015)
}

\author{
JimMY EMMANUEL RAMOS VALENCIA
}

\section{RESUMEN:}

El presente documento tiene como objetivo analizar las políticas públicas de combate a la dispersión e incremento de las enfermedades crónicas no transmisibles (ECTN) en México del año 2001 hasta el 2015. Tal objetivo plantea mostrar que la incidencia de la problemática de salud requiere que las reformas estructurales incidan en las políticas públicas para el combate del incremento de las enfermedades crónicas no transmisibles. La elección de las enfermedades responde a los llamados de atención de los organismos internacionales hacia los países de altos y medianos ingresos ante el incremento de la dispersión de la problemática de salud en el mundo. El artículo es una reflexión que pugna por ir más allá del análisis de los indicadores médicos y económicos que deben contrastarse con la información cualitativa recabada con las herramientas del método etnográfico. La propuesta del diseño de estrategias de evaluación desde el método etnográfico es para aportar información sobre los procesos de las dinámicas de combate implementadas para contar con información de las experiencias y narrativas, tanto de los enfermos, como de todo el personal de salud encargado de implementarlas.

\section{Palabras clave:}

Políticas Públicas, Método Etnográfico, Enfermedades Crónicas No Transmisibles, Información Cualitativa

\section{ABSTRACT:}

The aim of this document is to analyze public policies to combat the spread and increase of chronic-degenerative diseases in Mexico from 2001 to 2015. This aims to show that the growth of health problems requires structural reforms to affect 
in public policies to combat the increase of chronic diseases. The choice of diseases responds to calls from international organizations for high and middle-income countries in the face of an increase in the spread of health problems in the world. The article is a reflection that strives to go beyond the analysis of medical and economic indicators with the goal of qualitative information collected based on the tools of the ethnographic method. The proposal of the design of evaluation strategies from the ethnographic method is to give information on the processes of combat dynamics implemented to have information on the experiences and narratives, both patients and of all the health staff in charge of implementing them.

\section{Keywords:}

Public Policies, Ethnographic Method, Chronic Noncommunicable Diseases, Qualitative Information

\section{LA CONFORMACIÓN DE UNA POLÍTICA PÚBLICA}

En la comprensión las acciones y omisiones de un Estado con respecto a ciertas problemáticas es menester entender que ésta debe ser exigida y definida por la sociedad, ${ }^{1}$ es decir, requiere del llamado de atención de posturas de la sociedad con respecto a una determinada problemática. Toda problemática cuando adquiere un carácter colectivo exige la participación e implicación de los poderes públicos porque afecta a varios actores a tal punto que no es posible resolverlas solo con la interacción entre los interesados, por lo que la sociedad requiere que el Estado sea el encargado de analizar el contexto del problema y con base en tal análisis gestionar escenarios para su resolución. ${ }^{2}$ Las propuestas de resolución conforman una política pública en la cual son conformadas un:

... conjunto de medidas e intervenciones que se impulsan desde el Estado, que tienen como objetivo mejorar la calidad de vida de la población y lograr crecientes niveles de integración social, habilitando a los grupos socialmente excluidos, en las diversas dimensiones en que se expresa su exclusión (económica, política, territorial y sociocultural). La Política encuentra su expresión concreta en programas y éstos, a su vez, en proyectos. ${ }^{3}$

En este sentido, una política pública parte de la hipótesis de aplicar un conjunto de medidas e intervenciones factibles para lograr una situación de mejora. Sin embargo, esto no quiere decir que el nuevo escenario resuelva todos los problemas, pero al menos puede incrementar una mejor situación para los actores implicados. ${ }^{4}$

1 CHARLES ELDER, "El estudio de las políticas públicas; la hechura de las políticas; problemas públicos y agenda de gobierno", Problemas Públicos y Agenda de Gobierno, 3a. ed., edición de Luis F. Aguilar Villanueva, México, Porrúa, 2009, p. 51.

2 MAURICIO OLAVARRÍA GAMBI, Conceptos Básicos en el Análisis de Políticas Públicas. Ecuador, FLACSO, 2007, pp. 11 y 12.

3 Ministerio de Planificación y Cooperación División Social, Documento $N^{\circ}$ 4. Metodología de Análisis de Políticas Públicas: Conceptos y Criterios, Chile, Sistema Integrado de Evaluación de Intervenciones Públicas, 2000, pp. 9 y 10.

4 Nelson, Barbara y Lindenfeld, Thomas, "La formación de una agenda. El caso de maltrato a los niños", Problemas Públicos y Agenda de Gobierno, 3a. ed., edición de Luis F. Aguilar Villanueva, México, Porrúa, 2009, pp. 107 y 108. 
Y en la conformación de toda política pública siempre está presente la premisa de que las problemáticas son desplazadas y el ejercicio de intervención es el seguimiento de las estelas que conlleva tal desplazamiento. Porque muchas veces las políticas públicas son 'blancos nebulosos en movimiento', ya que con frecuencia los objetivos generales, el paquete consecuente de acciones y las relaciones entre ambos no se pueden ver con claridad y además se están moviendo. La mayoría de las veces las políticas públicas son ejercicios de exploración y aprendizaje constantes. Esto se debe a que: a) casi siempre hay una cierta incertidumbre en los procesos de formulación y ejecución de las mismas, lo que lleva a una retroalimentación permanente entre las diferentes "etapas" de las políticas públicas, y b) su contexto cambia frecuentemente. ${ }^{5}$

En la definición de los problemas públicos para insertarlos en la agenda ${ }^{6}$ entran en juego criterios de valoración entre el "deber ser" y el "es". Los actores, tanto los afectados, como los encargados de definir la problemática, parten de su estar en el mundo para la conformación de sus criterios y prioridades. A partir de tal clasificación prioritaria es que los actores toman las decisiones que correspondan a una resolución capaz de incorporar en gran medida el espectro de resolución del problema. Por ello, en la conformación de la agenda pública existe un proceso de incorporación normativa en los análisis; y para que tal proceso pueda adquirir el carácter normativo es menester contar con la evidencia empírica que sirva como base a toda política pública. "Por formación de la agenda se entiende el proceso a través del cual ciertos problemas o cuestiones llegan a llamar la atención seria y activa del gobierno como posibles asuntos de política pública".?

La base empírica que sirve para definir la problemática, identificar y definir los actores, y diseñar las estrategias de acción es lo que brinda la operacionalidad de la política. La operacionalidad es lo que permite avanzar en una dirección adecuada para la resolución del problema, y al mismo tiempo brinda la temporalidad de las propuestas de acción y resolución. La temporalidad de las estrategias brinda un carácter operativo del discurso implementado para solucionar el problema identificado y de tal manera evitar que solo sea una utopía.

Lo anterior sirve en el diseño de políticas públicas para identificar los recursos, capacidades y actores que permitan gestionar alternativas para solucionar los problemas. El proceso de definición de una problemática es importante porque:

En primer lugar, la capacidad de atención del gobierno es necesariamente limitada: siempre hay más asuntos por atender que tiempo para considerarlos. En segundo lugar, los problemas de políticas públicas no son a priori, sino resultados de definiciones. Si una situación específica o un conjunto de circunstancias constituyen un

5 José LUIS MÉNDEZ MARTíneZ, Análisis De Politicas Públicas: Teoría y Casos, México, Colegio De México, 2015.

6 Para conocer de manera integral el proceso de conformación de la agenda pública se recomienda consultar Luis Aguilar Villanueva, "La formación de la agenda", Problemas públicos y agenda de gobierno, México, Porrúa, 2009, pp. 21-51.

7 CHARLES ELDER y ROGER COBB, "Formación de la agenda. El caso de la política de los ancianos", Problemas públicos y agenda de gobierno, México, Porrúa, 2009, p. 77. 
problema y, por tanto, son un asunto capaz de despertar la preocupación del público, depende no sólo de los hechos sino también de las creencias y valores. ${ }^{8}$

Por lo tanto, construir alternativas de solución desde el punto de vista de las políticas públicas siempre es con base en los recursos y capacidades que los operadores y gestores políticos tienen a su disposición; conciliando en todo momento en no transgredir valores sociales, históricos o culturales, y evitando en todo momento del partir de definiciones normativas. Sin embargo, no hay que perder de vista que en la conformación de una política pública entran en juego luchas de poder económico, político y epistemológico que confronta a los actores, que presiona al Estado en la toma de decisiones y en la conformación de la agenda de prioridades. ${ }^{9}$

\section{EL MÉTODO ETNOGRÁFICO PARA EL ANÁLISIS DE UNA POLÍTICA PÚBLICA}

El discurso biomédico como institución y práctica social es un poderoso aparato ideológico para crear e imponer la subjetividad del enfermo, el estar, ser y sentirse enfermo. De acuerdo con la gravedad de la patología es que éste asume una identidad y papel asignado. El enfermo puede ser consciente del papel que el discurso biomédico (la institución) le ha asignado y a partir de ello comenzar un proceso de resistencia para explicar con su voz el proceso de interpelación al que está sometido, un proceso mediante el cual deja de escuchar al médico y comienza a buscar otras voces, una de ellas la suya. Y es en este momento de interiorización de la enfermedad cuando el enfermo en la búsqueda de otras "voces", que muchos de los cuidados y tratamientos respecto a una enfermedad sufren un giro inesperado; que en lo que respecta a enfermedades que requieren tratamientos prolongados, pueden determinar el seguimiento, o no, y el éxito, o no, esperado. ${ }^{10}$

Es en este proceso de interiorización y comprensión del discurso biomédico cuando escuchar al enfermo brinda información pertinente para conocer la dimensión e interiorización de las estrategias de atención, prevención y tratamiento transmitidas a los enfermos. Es este momento donde el método etnográfico adquiere un valor porque en las narrativas de enfermedad es donde salen a la luz los episodios del proceso que marcaron el proceso de cuidado y atención de los pacientes. ${ }^{11}$ Es por ello, que la descripción, abstracción y análisis de estos procesos son los que brindan la oportunidad de brindar soluciones integrales a complejas problemáticas de salud. ${ }^{12}$

8 Ídem.

9 LUIS AGUILAR VILLANUEVA, Problemas Públicos...cit., pp. 52 y 53.

10 DiGiacomo, Susan, "Autobiografía crítica y teoría antropológica. Reflexiones en torno a la enfermedad y la identidad cultural y profesional", en JOSEP COMELLES; MARIA ANTONIA MARTORELL POVEDA y MARIOLA BERNAL, Antropología y enfermería. Campos de encuentro. Un homenaje a Dina Garcés, España, Universitat Rovira i Virgili, 2009.

11 GEORGE MURDOCK, "Anthropology and Its Contribution to Public Health", American journal of public health and the nation's health, 1952, 42(1), pp. 7-11.

12 Ídem. 
En la atención integral de una enfermedad, el paciente inicia una búsqueda en la cual requiere identificar, interpretar y comprender las categorizaciones culturales que la conforman. Para esto, en su análisis pugna por conocer: el aspecto biológico, Disease; las vivencias subjetivas, lllness; y las simbolizaciones socioculturales, Sickness. ${ }^{13}$ En conjunto, las tres dimensiones son las que le brindan un panorama general de la enfermedad que le acongoja, y a partir de sus reflexiones $\mathrm{y}$ juicios, es que pone en práctica estrategias para afrontar el padecimiento. ${ }^{14}$ Estas tienen un carácter social y cultural, que las diferencia a partir de la forma en que son estructuradas y diferenciadas acorde con el contexto sociocultural del grupo de riesgo. Por ello, para comprender integralmente la dispersión de una enfermedad es menester conceptualizarla como un hecho social respecto del cual las sociedades construyen acciones, técnicas e ideologías. ${ }^{15}$

Integrar las interpretaciones de las experiencias de enfermedad sirve para formular los conceptos de salud y atención, entendidos como eventos simbolizados por los grupos de riesgo; así como también, categorizar sus prácticas y discursos preventivos. Tal método de análisis cualitativo y social en salud ilustra las formas en que las personas expuestas a una enfermedad requieren y recurren a prácticas simbolizadas que les permitan restablecer su estado físico y emocional; es decir, que le provean salud. ${ }^{16}$ Comprender la lógica de tales prácticas y representaciones sociales de la enfermedad es factible a través del análisis de las narrativas de la enfermedad. ${ }^{17}$

En las narrativas de la enfermedad entran en juego todas las estrategias que las personas utilizan para mantener su salud, por ello, este discurso sobre la enfermedad es lo que nos permite conocer el grado de integración de las políticas desde el punto de vista del actor, es decir, de la población objetivo a la que es destinada, de tal manera que permite un acercamiento desde el cual resulta factible proponer alternativas de base para los programas gubernamentales. ${ }^{18}$ Desde esta perspectiva, los resultados de la investigación de temas de procesos y políticas públicas tienen un carácter crítico desde la mirada del beneficiario, otorgando con ello la ventaja de que las propuestas críticas de los procesos de políticas públicas brindan la voz al actor, al beneficiario, que es el principal interesado en que las estrategias propuestas sean de lo más integrales para el cuidado y atención de su salud. ${ }^{19}$

13 ARTHUR KLEINMAN, Patients and healers in the context of culture: An exploration of the borderland between anthropology, medicine, and psychiatry, Estados Unidos, Univ of California Press, 1981.

14 ARTHUR KLEINMAN, The illness narratives: Suffering, healing, and the human condition, Estados Unidos, Universidad de Michigan, 1988.

15 EDUARDO MENÉNDEZ, “La enfermedad y la curación ¿Qué es medicina tradicional?”, Alteridades, 1994, 4 (7), 71-83, p. 79.

16 MARC MICOZZI, "Culture, anthropology, and the return of "complementary medicine"”, Med Anthropol Q, 2002, Dec;16(4), pp. 398-403.

17 ARTHUR KLEINMAN, op., cit.

18 ROBERT HEIGHTON y CHRISTY HEIGHTON “Applying the Anthropological Perspective to Social Policy”, ELIZABETH EDDY y WILLIAM PARTRIDGE (eds.), Applied Anthropology in America, Estados Unidos, 1987, pp. 390-430.

19 CYRIL BELSHAW, The Sorcerer's Apprentice: An Anthropology of Public Policy, New York, Pergamon Press, 1976. 
El método etnográfico en profundidad para describir las condiciones de vida, la organización social, las prácticas distintivas, los valores y las creencias del grupo social contribuye con su enfoque centrado en las personas al diseño de políticas cuyo punto de partida esté orientado a cómo las personas experimentan las políticas públicas y cómo estas políticas impactan sus vidas. ${ }^{20}$ En gran medida, el principal propósito del método etnográfico para el análisis de la política pública es proporcionar información a los tomadores de decisión como apoyo y complemento de los indicadores macro sobre el proceso de implementación y evaluación de las políticas públicas. Lo interesante de este enfoque es que parte de las personas y sus resultados están destinados a ellas. ${ }^{21}$

El poder crítico de la etnografía consiste en la precisión de su descripción empírica, que puede usarse para contradecir las representaciones del mundo social basadas en las creencias e ideologías sociopolíticas dominantes. Darle voz a los destinatarios de las políticas públicas, como un medio para empoderarlos, brindando un complemento útil a la historia y la sociología de la construcción de los problemas públicos para redefinir el panorama social, explotar las categorías recibidas, explorar las experiencias subjetivas y exponer sus puntos de vista sobre sus problemáticas que muchas veces difieren de los puntos de vista oficialmente aceptados. ${ }^{22}$

Una etnografía crítica de la política pública consiste en yuxtaponer los resultados de la observación etnográfica a las propuestas de los responsables de las políticas para romper con los discursos oficiales y mucha de la retórica transmitida por los medios. Los métodos de trabajo de campo cualitativos se distinguen de otras perspectivas de análisis de las políticas por el hecho de brindar voz a la población a la que son destinadas las acciones de la política, es decir, a los beneficiarios. En la práctica esta perspectiva de análisis proporciona información útil a los tomadores de decisión con base en un enfoque crítico que parte de la reflexión sobre los contextos, prácticas y usos sociales y políticos de la investigación en ciencias sociales. ${ }^{23}$

El desafío de los enfoques positivistas de la política pública; la confrontación con el sentido común y las opiniones oficiales sobre la política; y la descripción y análisis de las experiencias individuales con base en las observaciones micro brinda una perspectiva más amplia de las estructuras de poder y desigualdad. Tales resultados revelan los procesos de dominación social, económica, simbólica y política que operan a través de la implementación de políticas públicas. ${ }^{24}$

20 DAVE CAMPBELL, “Anthropology's Contribution to Public Health Policy Development”, McGill Journal of Medicine, 2010, 13(1)1, pp. 76-83.

21 MELISSA PARKER y IAN HARPER, “The Anthropology of Public Health", Journal of Biosocial Science, 2006, 38(1), 1-5, doi:10.1017/S0021932005001148

22 Ídem.

23 JOAO BIEHL y ADRIANA PETRYNA, “Critical Global Healht”, When People Come First. Critical Studies in Global Health, Estados Unidos, Princeton University Press, 2013, pp. 1-20.

24 VINCENT DUBOIS, “Critical policy ethnography”, en Fischer, Frank, Torgerson, Douglas, Durnová, Anna y Orsini, Michael (eds.), Handbook of Critical Policy Studies, Estados Unidos, Edward Elgar Publishing, 2017, pp. 462-480. 


\section{PANORAMA DE LAS ENFERMEDADES CRÓNICO NO TRANSMISIBLES (ECTN) EN MÉXICO}

En las propuestas federales en materia de salud de México desde la primera década del siglo XXI ("Plan Nacional de Salud 2001-2006) "La democratización de la salud en México. Hacia un sistema universal de salud", "Programa Sectorial de Salud 2007-2012. Por un México sano: construyendo alianzas para una mejor salud", "Programa Sectorial de Salud 2013-2018", los diagnósticos de los que parten muestran que los principales retos que enfrenta el sistema de salud son las enfermedades crónicas no transmisibles (ECTN) porque debido a su incidencia y prevalencia en la población, tanto en la morbilidad como en la mortalidad, los análisis diacrónicos y prospectivos muestran un incremento - desde la década de los ochentas - a tal grado que caracterizan el perfil epidemiológico del país. ${ }^{25}$

En lo que respecta a la administración de los países estas enfermedades son una problemática de salud porque son la causa más frecuente de incapacidad prematura, y dado a su complejidad - en su prevalencia entran en juego factores genéticos, sociales, económicos, culturales e individuales - son un gasto continuo debido a la temporalidad que implica su tratamiento (de mediano a largo plazo), así como también por sus altos costos, además de que estas enfermedades requieren monitoreo constante y especializado, así como tratamientos a largo plazo y el sistema de salud mexicano no fue diseñado para este fin, lo cual se manifiesta en servicios insuficientes para tratar a los pacientes. ${ }^{26}$

Por ello, para revertir la incidencia de la enfermedad es menester poner en práctica estrategias institucionales en las que haya interacción y retroalimentación en las dimensiones sociales e individuales para lograr modificaciones en los patrones y estilos de vida. Es decir, requiere de políticas públicas que cuenten con estrategias integrales por medio de las cuales la población adquiera una cultura de la prevención a partir de iniciativas institucionales. Tales políticas son propuestas con base en la premisa del enfoque institucional que pugna por una visión en la cual las instituciones son las "...crea[doras de] conductas, [debido a que] la estructura crea cultura y no a la inversa" y por ello no perder de vista que la problemática no puede ser resuelta sólo con las acciones individuales. ${ }^{27}$

Las evaluaciones e indicadores sobre la incidencia y prevalencia de las ECNT que utiliza la Secretaría de Salud para proponer las estrategias y políticas públicas referidas a salud en México son: "Boletín de información estadística de la Secretaría de Salud"; "Proyecciones de la Población e Indicadores Demográficos Básicos

25 Programa Nacional de Salud 2001-2006, Gobierno de la República, Secretaría de Salud, México, p. 99; Programa Sectorial de Salud 2007-2012, Gobierno de la República, Secretaría de Salud, México; Programa Sectorial de Salud 2013-2018, Gobierno de la República, Secretaría de Salud, México, pp. 24 y 49; SotoEstrada, Guadalupe, Moreno-Altamirano, Laura y Pahua Diaz, Daniel, "Panorama epidemiológico de México, principales causas de morbilidad y mortalidad", Revista de la Facultad de Medicina de la UNAM, Vol. 59, 6, Noviembre-Diciembre, 2016, pp. 11-19.

26 IMCO, Kilos de más, pesos de menos. Los costos de la obesidad en México, Instituto Mexicano para la Competitividad A.C.,2015, México.

27 ALICIA GUZMÁN-LEÓN, “Comparar para construir política pública en tiempos de globalización”, Revista Iberoamericana de Educación Superior, vol. VII, núm. 20, 2016, p. 154. 
de México a Nivel Nacional 2010-2050”; "Encuesta Nacional de Salud y Nutrición (ENSANUT )"; para los costos de la enfermedad los planes y programas parten de los análisis realizados a escala global para Latinoamérica en los informes sobre el "costo de la diabetes en Latinoamérica" para 2003 y 2015 respectivamente.

\section{PLAN NACIONAL DE SALUD 2001-2006 "LA DEMOCRATIZACIÓN DE LA SALUD EN MÉXICO. HACIA UN SISTEMA UNIVERSAL DE SALUD"}

En los albores del siglo XXI y con la entrada de un gobierno encabezado por un partido de oposición en México, los objetivos del "Plan Nacional de Salud 2001-2006" estuvieron enfocados en cambios de carácter estructural, en referencia a la asignación de los recursos, el papel de las administraciones locales (otorgar la mayor responsabilidad de la salud de la población a los Municipios) y el cambio en la relación médico-paciente, asignando una mayor responsabilidad en el cuidado de su salud al enfermo.

El llamado de atención sobre la emergencia en la prevalencia de las enfermedades está presente en el "Plan Nacional de Salud 2001-2006. La democratización de la salud en México. Hacia un sistema universal de salud". El plan parte de un enfoque que hace referencia al reto de la equidad y los daños a la salud; documenta el aumento de los decesos por ECTN que han pasado de un 44 a un $73 \%$ en el período $1950-2000^{28}$ y muestra una proyección que indica que para el 2025 habrá un aumento del $77.8 \% .{ }^{29}$ Ante tal panorama de morbilidad y mortalidad en México, el programa propone analizar las condiciones de salud de la población desde un enfoque que aborde la prevalencia de la enfermedad en la población desde una perspectiva que tome en cuenta la discapacidad generada por el nuevo panorama epidemiológico.

Para ello, el plan parte de la propuesta realizada por la Organización Mundial de la Salud (OMS) de indicadores integrales que sirvan para brindar un panorama que permita comprender el espectro de afectación de este conjunto de enfermedades en la población nacional. Tales indicadores son los de Esperanza de Vida Saludable (EVISA) y Años de Vida Saludable (AVISA). ${ }^{30}$ El primero sirve para medir los años con buena salud de una persona en una sociedad determinada y el segundo evalúa la merma en salud debido a la muerte prematura con los años que una persona vive con discapacidad (Perdida debida a Muerte Prematura más Pérdida debida a discapacidad igual a pérdida de salud). Como complemento de la información presentan estimaciones con base en la información proporcionada por la Secretaría de Salud que muestra que desde la década de los ochenta las ECNT concentran la mayor tasa de mortalidad en el país. ${ }^{31}$

28 Programa Nacional de Salud 2001-2006, Gobierno de la República, Secretaría de Salud, México, p. 32.

29 Ibidem, p. 33.

30 GABRIELA RODRÍGUEZ-ABREGO; JORGE ESCOBEDO DE LA PEÑA; BEATRIZ ZURITA G y TERESITA DE JESÚS RAMÍREZ, "Esperanza de vida saludable en la población mexicana con seguridad social”, Perinatol Reprod Hum, 2006; 20, pp. 4-18.

31 La presentación de resultados realizada por el Dr. Eduardo González Pier titulada "Impacto socioeconómico de las enfermedades crónicas no transmisibles" en la $30^{a}$ Reunión Anual del Instituto Nacional de Perinoto- 
El documento parte de la perspectiva teórica de la "transición epidemiológica" para analizar la escalada de las Enfermedades Crónico no Transmisibles en México. Esta propuesta teórica parte de la relación directa de las variables demografía, organización social y estructura económica con la salud. Este postulado teórico parte del supuesto evolutivo unilineal en las sociedades que las hace transitar de las enfermedades trasmisibles hacia los padecimientos no transmisibles. Las primeras asociadas a carencias primarias de los Estados para proporcionar a su población contextos integrales de nutrición, vivienda, educación y abastecimiento de agua; a un lapso en el cual, una vez superadas estas carencias las enfermedades de las poblaciones comienzan a relacionarse con factores genéticos y conductas destructivas. Además de que la "carga de enfermedad", muerte e invalidez pasa de los niños y jóvenes hacia los grupos de mayor edad. ${ }^{32}$ El PNS (20012006) muestra proyecciones sobre el incremento de la mortalidad a causa de las ECNT a un ritmo ascendente. Los riesgos de salud atribuidos al incremento de la carga de enfermedad son atribuidos a la industrialización y la urbanización, partiendo de la teoría de la transición epidemiológica. ${ }^{33}$

La propuesta del plan es la democratización de la salud ante los retos de la equidad, la calidad y la protección financiera. Democratizar la salud significa el acceso universal a los servicios de salud, estimular la participación de todos los actores de la sociedad y asumir la responsabilidad de su salud, influir en el diseño de la agenda y toma de decisiones. Entre líneas, en el discurso promulgado en este Plan Nacional de Salud comienza el giro epistemológico respecto a la responsabilidad integral de los actores tanto en mantener su salud, como en brindar propuestas para la agenda pública en esta materia y en la toma de decisiones del sector: "Estrategia 9. Ampliar la participación ciudadana y la libertad de elección en el primer nivel de atención". ${ }^{34}$

Respecto a las problemáticas asociadas con la transición epidemiológica el plan es claro al mencionar que el problema tiene un carácter multidimensional, y debido a esto es que las estrategias de política pública puestas en marcha deben incluir actores del sistema de salud, público o privado, de la sociedad civil, la empresa, los medios de comunicación y la educación; que coordinen su trabajo

logía "El origen perinatal de las enfermedades del adulto" disponible en: http://www.sidss.salud.gob.mx/ contenidos/PDF/Presentacion_INPER_EGP_220415_v2.pdf, los resultados de los análisis utilizan datos del The Institute for Health Metrics and Evaluation (IHME) que es un organismo conformado en el año 2007 partiendo de la Gobernanza en salud para proporcionar datos objetivos para la elaboración de políticas públicas acordes con las necesidades y problemáticas de salud de los países. Los datos de tal instituto son proporcionados por las secretarías y ministerios de salud de los países del mundo: The Institute for Health Metrics and Evaluation (IHME) launches with the goal of providing an impartial, evidence-based picture of global health trends to inform the work of policymakers, researchers, and funders. Main supporters are the Bill \& Melinda Gates Foundation and the state of Washington, disponible en: http://www.healthdata.org/ about/history.

32 JULIO FRENK, et al., "La transición Epidemiológica en América Latina”. Bol of Sanit Panam, 1991, 111(6); Vera Bolaños, M. (2000) "Revisión crítica a la teoría de la transición epidemiológica”, Papeles de Población, vol. 6, núm. 25, julio-septiembre, 2000. 
para implementar estrategias de salud enfocadas en la prevención de riesgos asociados con las enfermedades:

Los problemas emergentes, por su parte, se deben en su mayoría a la creciente exposición a estilos de vida que dañan la salud. El sedentarismo, el consumo de tabaco y alcohol, la inseguridad pública y vial, la violencia en el hogar, y el deterioro de las redes familiares y sociales han crecido incesantemente a lo largo del último medio siglo en nuestro país. Su control depende de la implantación de estrategias anticipatorias y preventivas que incidan sobre los hábitos de vida. ${ }^{35}$

En lo que respecta a mortalidad en el período abordado, la diabetes mellitus y la cardiopatía isquémica generan individualmente una gran parte del peso de la enfermedad en el país. ${ }^{36}$ Ambos padecimientos están asociados con conductas de riesgo, así como también tienen una relación directa con enfermedades como la tuberculosis, pero lo más importante, es la carga de enfermedad ${ }^{37}$ que representan para el país. Estas enfermedades comparten algunos factores de riesgo que es esencial atender, destacan dentro de ellos el sobrepeso y la obesidad. ${ }^{38}$ Por ello las acciones propuestas para combatir el sobrepeso y la obesidad en el programa son: Promover la actividad física y las dietas bajas en calorías; proporcionar a la población información sobre la obesidad y las enfermedades crónicas asociadas a ella; regular estrictamente la promoción publicitaria de los alimentos ricos en calorías, incorporando a la publicidad información sobre los riesgos asociados al consumo de estos productos; implantar estrategias para la identificación de factores de riesgo y para el diagnóstico temprano de las enfermedades relacionadas con el sobrepeso y la obesidad. ${ }^{39}$

Ante el predominio de las ECTN y las lesiones (accidentales y violentas) que caracteriza el perfil epidemiológico del país, cuya particularidad es que son rezagos que no están concentrados en algún decil de ingresos de la población, la administración postula una estrategia para "Enfrentar los problemas emergentes mediante la definición explícita de prioridades", ${ }^{40}$ partiendo de la premisa de que contrarrestar la proliferación y dispersión de las ECNT en la población requiere la promoción de estilos de vida saludables y estrategias de difusión de información que concientice a la población sobre la importancia de la detección temprana de este grupo de padecimientos.

35 Ibidem, p. 27.

36 Ibidem, pp. 44 y 45.

37 Sobre el cálculo de la carga de enfermedad se recomienda consultar: NATALIA SÁNCHEZ DÍAZ, "La carga de la enfermedad", Revista Colombiana de Psiquiatría, XXXIV (2),2005, pp. 298-304.

"Sobrepeso y obesidad. México presenta una de las prevalencias de sobrepeso y obesidad más altas del mundo", Plan Nacional de Salud 2001-2006, p. 51.

40 Ibidem, pp. 99-101. 


\section{SEXENIO 2007-2012, PROGRAMA NACIONAL DE SALUD "POR UN MÉXICO SANO: CONSTRUYENDO ALIANZAS PARA UNA MEJOR SALUD"}

Durante este sexenio la inversión en salud centra su atención en estrategias de prevención para abatir la "carga de enfermedad"41 (Sánchez Díaz 2006: 300 y 301) de las ECTN. Prueba de ello es la puesta en marcha en febrero de 2007 de la "Estrategia Nacional de Prevención y Promoción para una mejor salud". Esta estrategia hace un llamado a los especialistas en salud y a las instituciones para capacitar al personal de salud para afrontar el nuevo perfil epidemiológico. ${ }^{42} \mathrm{La}$ estrategia hace énfasis en la necesidad de poner en práctica políticas públicas de salud dirigidas a la población para conformar una cultura de la prevención que sirva para conformar patrones de estilos de vida saludables. ${ }^{43}$

El cambio de visión por parte de la administración 2006-2012 para el control de las ECNT fue como respuesta a las evaluaciones de la administración previa cuyos resultados obtenidos por las estrategias planteadas arrojó que invertir en los tratamientos desencadenó resultados negativos debido a que un adecuado control de las ECTN "Implica un proceso educativo para entender la enfermedad, cambios significativos y focalizados en las conductas, utilización a largo plazo de múltiples fármacos y evaluaciones frecuentes, además de la participación de especialistas en conjunto con la familia y la comunidad". ${ }^{44}$

Para el seguimiento de las acciones encaminadas a reducir la mortalidad por el conglomerado de ECNT en esta administración fue puesta en marcha la "Estrategia Nacional de Promoción y Prevención para una Mejor Salud. La salud, tarea de todos" en junio de 2007. Dicha estrategia tuvo como una de sus líneas de acción fundamentales "Construir una Política pública a favor de la salud". El hilo conductor de la estrategia fue la misma problemática identificada en el sexenio anterior: el incremento de la mortalidad y las hospitalizaciones a causa de las ECNT $^{45}$ (Secretaría de Salud, Estrategia Nacional de Promoción y Prevención para una Mejor Salud: 31-39).

La "Política Pública a Favor de la Salud" propuesta planteó como objetivo general: "Disminuir mediante acciones anticipatorias, el impacto que tienen las enfermedades y las lesiones sobre individuos, familias, comunidades y la sociedad en su conjunto". ${ }^{46}$ Fue planteado un decálogo estratégico para "disminuir el impacto de

\footnotetext{
41 NATALIA SÁNCHEZ DIAZ, "La carga de..., cit., p. 300.

42 "Síndrome metabólico: definición y panorama epidemiológico en México", Plan Nacional de Salud 20072012, p. 20.

43 Secretaría de Salud, "Acuerdo por el que se emiten las Reglas de Operación e Indicadores de Gestión y Evaluación del Programa Comunidades Saludables", Diario Oficial de la Federación (DOF), martes 30 de diciembre de 2008 (Decimosegunda Sección) http://www.dof.gob.mx/nota_to_doc.php? codnota $=5326217$ JOSÉ ÁNGEL CÓRDOVA-VILLALOBOS et. al., "Las enfermedades crónicas no transmisibles en México: sinopsis epidemiológica y prevención integral”, Salud pública Méx, vol.50, n.5, 2008, pp. 420 y 421.

45 Estrategia Nacional para la Prevención y el Control del Sobrepeso, la Obesidad y la Diabetes (2013), pp. 31-39.

46 "Estrategia Nacional de Promoción y Prevención para una Mejor Salud: 10 componentes. Esta estrategia tiene como objetivo disminuir el impacto de las enfermedades y las lesiones en individuos, familias y comu-
} 
las enfermedades y las lesiones en individuos, familias y comunidades mediante intervenciones específicas dirigidas a las personas que se combinarán con intervenciones dirigidas a la construcción de entornos favorables a la salud". ${ }^{47}$

La puesta en marcha de la estrategia nacional fue indispensable para atender los riesgos relacionados con las ECTN como una forma de abordar y controlar algunos de los riesgos a través de la promoción de la salud y fomentando comportamientos relacionados con determinantes positivos críticos como: la alimentación, la higiene y la actividad física, cuyo deterioro o descuido es común a varias de las principales causas de daños a la salud en el país. Debido a que el sobrepeso y la obesidad ${ }^{48}$ es el fenómeno que detona que las principales causas directas e indirectas de daños a la salud (diabetes, enfermedades cardiovasculares, enfermedad cerebro-vascular, dislipidemias e hipertensión) como resultado de los factores de riesgo (falta de actividad física y nutrición inadecuada) que es indispensable combatir y controlar a través de campañas de promoción de conductas saludables y control de riesgos específicos.

Con el fin de brindarle un carácter transversal a la política la estrategia partió de la premisa de que todas las acciones requieren de la cooperación interinstitucional e intersectorial para colaborar en la conformación de la promoción de la salud de tal manera que incida en promover en la población el conocimiento de los determinantes de la salud y los entornos saludables. De tal forma fueron planteados los actores y sus responsabilidades para integrar a la salud en las políticas públicas de otros sectores. ${ }^{49}$

Al finalizar el sexenio ${ }^{50}$ la prevalencia de sobrepeso y obesidad en menores de cinco años registró un ligero ascenso, un $12 \%$ en la región norte, representando

nidades mediante intervenciones específicas dirigidas a las personas que se combinarán con intervenciones dirigidas a la construcción de entornos favorables a la salud”, Plan Nacional de Salud 2007-2012, México, pp. 94 y 95 .

47 Determinantes favorables a la salud: Impulsar el consumo de alimentos con baja densidad energética, bajo contenido de grasa animal y bajo contenido de sal; adecuada higiene personal y saneamiento básico: Incremento en la actividad física; equidad inmunológica: vacunación; reducción del consumo de tabaco (activo y ajeno); consumo sensible y no riesgoso de bebidas alcohólicas; reducción del consumo de drogas ilegales y drogas médicas no prescritas; sexualidad responsable y protegida (anticoncepción); vialidad Segura; factores psicosociales; acceso garantizado al paquete de servicios de promoción y prevención para una mejor salud (incluye detección oportuna y tratamiento); roles y relaciones equitativas de género, pobreza, migración, entre otras. Entornos favorables a la salud: Viviendas; escuelas; sitios de trabajo (incluyendo albergues de jornaleros); unidades de salud; transportes; espacios de esparcimiento; municipios; ciudades. Estrategia Nacional de Promoción y Prevención para una Mejor Salud, México, 2007, p. 45.

48 Seis de cada diez mexicanos tienen alguna forma de dislipidemia - Medscape - 23 de nov de 2018. Recuperado de https://espanol.medscape.com/verarticulo/5903409\#vp_3

49 En la "Estrategia Nacional de Promoción y Prevención para una Mejor Salud" pp. 65-70 están descritas las acciones que corresponden a cada institución.

50 En el "Análisis del cumplimiento de los indicadores de resultados de la Secretaría de Salud 2007-2012" el indicador de resultados del programa de cobertura de detección de diabetes mellitus cumplió con las metas planteadas. Metas dirigidas a la detección de personas mayores de 20 años que potencialmente estén en la escala de riesgo. El indicador sólo menciona que fue cumplida la meta (sobrepasada de hecho) y que las personas recibieron orientación a partir de un compendio de manuales y guías para el cuidado de su salud. A lo largo de la evaluación no es mencionado algún otro indicador sobre el tema, incluso la página citada en el documento como una guía para informarse sobre la diabetes ya no está en funcionamiento (CONEVAL, 
alrededor de 5664870 niños con sobrepeso y obesidad en el ámbito nacional para el año 2012. Con respecto a la población adolescente del país, más de una tercera parte tiene exceso de peso (35\%); la prevalencia combinada de sobrepeso y obesidad en adultos fue de $71.28 \%$. La obesidad fue más alta en el género femenino que en el masculino, mientras que el sobrepeso fue mayor en el género masculino. También, la prevalencia de obesidad presenta diferencias por nivel socioeconómico: es mayor en el alto que en el bajo, al igual que en las zonas urbanas en comparación con las rurales y en la región norte del país en comparación con el sur y el centro. ${ }^{51}$

\section{SEXENIO 2013-2018, PROGRAMA SECTORIAL DE SALUD, "CIMENTAR LAS BASES DE UN SISTEMA DE SALUD UNIVERSAL"}

En el plan de salud de esta administración de nuevo el llamado de atención indica el crecimiento de las Enfermedades Crónico no Transmisibles (ECTN), a pesar de las estrategias puestas en marcha en las anteriores administraciones. ${ }^{52}$ Ante la escalada de la dispersión y crecimiento de la enfermedad en el plan se hace mención del factor de riesgo asociado a ellas: el sobrepeso y obesidad, que determinan el padecimiento de diabetes (tipo II) e hipertensión en la población del país.

El análisis sobre el panorama epidemiológico está construido a partir de la evolución de la enfermedad con base en los resultados de la Encuesta Nacional de 1993 donde se identificó una prevalencia nacional del Síndrome Metabólico de aproximadamente $30 \%$ en la población de 20 años y más; así como también los resultados de La Encuesta Nacional de Salud 2000 que mostró que cerca de $8 \%$ de los adultos mayores de 20 años sufren de diabetes tipo II; y aproximadamente $30 \%$ de hipertensión arterial (con una prevalencia de dislipidemia cercana a 30\%). Y, por último, los datos de la Encuesta Nacional de Salud y Nutrición 2006 y 2012 que indican que siete de cada diez personas adultas presentan sobrepeso y obesidad. ${ }^{53}$

Ante tal panorama la prospección de los datos e información de la creciente epidemia de enfermedades crónicas en México, asociadas a la obesidad, como la diabetes y la hipertensión arterial, anticiparon para el sexenio un desarrollo explosivo del Síndrome Metabólico. Y por ello las cifras plantearon a la administración el desarrollo de estrategias de control de los riesgos asociados al

Análisis del cumplimiento de los indicadores de resultados, Secretaría de Salud, 2007-2012: 16). La página para que la población pueda informarse sobre la diabetes www.todoendiabetes.org remite ahora a la página www.silanes.com.mx que es un corporativo médico y farmacéutico.

51 JAVIER DÁVILA-TORRES; JOSÉ DE JESÚS GONZÁLEZ-IZQUIERDO y ANTONIO BARRERA-CRUZ, "Panorama de la obesidad en México", Rev Med Inst Mex Seguro Soc., 53(2), 2015, pp. 240-9.

52 Plan Nacional de Desarrollo 2013-2018, Gobierno de la República, Programa Sectorial de Salud. México, pp. 25-30.

53 JUAN GUTIÉRREZ; JUAN RIVERA; TERESA SHAMAH; CARLOS OROPEZA y MAURICIO HERNÁNDEZ ÁVILA, Encuesta Nacional de Salud y Nutrición 2012, Instituto Nacional de Salud Pública, México, 2012, Disponible en https://ensanut.insp.mx/informes/ENSANUT2012ResultadosNacionales.pdf 
síndrome, así como también, para capacitar al personal de salud para la atención de las personas afectadas, cuyas prospecciones indican un aumento importante en las décadas por venir. ${ }^{54}$

De igual manera que en las anteriores administraciones la guía del plan con respecto a las ECTN hace énfasis en la transición epidemiológica que vive el país, asociada con el envejecimiento de la población y al creciente desarrollo de riesgos relacionados con estilos de vida poco saludables (mala nutrición, alto consumo de calorías y grasas de origen animal, bajo consumo de frutas y vegetales, el sedentarismo y el consumo de sustancias adictivas ${ }^{55}$ (Diagnóstico General de la Salud Poblacional 2015: 128-134).

Debido a la dispersión de los estilos de vida poco saludables, el plan propone estrategias que son responsabilidad del Estado para el desarrollo y fortalecimiento de una cultura de la salud que enfatice la importancia de la adopción de estilos de vida saludables, fomente el autocuidado de la salud e impulse la prevención de la enfermedad y las discapacidades. Para lograr mejores condiciones de salud en general y enfrentar menores cargas de enfermedad, para que la población evite exponerse al sobrepeso y la obesidad porque son causas de empobrecimiento económico debido a que inciden negativamente en su productividad laboral y además provocan gastos elevados en salud debido a su relación con el desarrollo de ECNT (gastos individuales, los gastos del bolsillo y al Estado debido a los costos de los tratamientos).

El sexenio propone la "Estrategia Nacional para la Prevención y el Control del Sobrepeso, la Obesidad y la Diabetes" (ENPCSOD) para construir una política pública nacional que genere hábitos de consumo alimenticio saludables, y actividad física en la población. Al igual que en administraciones previas la estrategia hace énfasis que es menester involucrar, a los sectores público y privado y la sociedad civil. El objetivo general planteado es mejorar los niveles de bienestar de la población y contribuir a la sustentabilidad del desarrollo nacional al desacelerar el incremento en la prevalencia de sobrepeso y obesidad en los mexicanos, a fin de revertir la epidemia de las enfermedades no transmisibles, particularmente la diabetes mellitus tipo 2, a través de intervenciones de salud pública, un modelo integral de atención médica y políticas públicas intersectoriales. La estrategia involucra los marcos de acción de "Promoción a la salud" (promoción de hábitos saludables), "Prevención de enfermedades" (detección temprana y sistema de monitoreo), "Atención Médica" (capacitación del personal de salud en prevención y control de enfermedades), "Regulación sanitaria y política fiscal".

En lo que respecta a los resultados del análisis con referencia a la salud pública la estrategia brinda un informe del panorama de la salud en México que responde al incremento del sobrepeso en la población atribuido al:

\footnotetext{
54 Subsecretaría de Integración y Desarrollo del Sector Salud. Dirección General de Evaluación del Desempeño, Informe sobre la Salud de los Mexicanos. Diagnóstico General de la Salud Poblacional, 2015, Disponible en www.dged.salud.gob.mx/contenidos/dedss/issm2015.html

55

Ibidem, pp. 128-134.
} 
...fenómeno de transición nutricional que experimenta el país, teniendo como características una occidentalización de la dieta, en la cual 1) aumenta la disponibilidad a bajo costo de alimentos procesados, adicionados con altas cantidades de grasas, azúcares y sal; 2) aumenta el consumo de comida rápida y comida preparada fuera de casa para un sector creciente de la población; 3) disminuye el tiempo disponible para la preparación de alimentos; 4) aumenta de forma importante la exposición a publicidad sobre alimentos industrializados y de productos que facilitan las tareas cotidianas y el trabajo de las personas, disminuyendo de este modo su gasto energético; 5) aumenta la oferta de alimentos industrializados en general; y 6) disminuye de forma importante la actividad física de la población. ${ }^{56}$

Ante tal panorama las acciones a realizar para la prevención y control del sobrepeso, la obesidad y la diabetes involucran los distintos marcos de acción de la salud, específicamente la Promoción de la salud: acciones para el desarrollo de aptitudes personales para la salud (promoción de alimentación correcta), desarrollo de entornos saludables (escuela y sitios de trabajo), acciones comunitarias (actividades en espacios públicos) e impulso de políticas públicas saludables (acción de vigilancia en alimentos y bebidas, medios masivos de comunicación, orientar al consumidor y establecer medidas fiscales para reducir el consumo de alimentos de alta densidad energética y escaso valor nutrimental).

Para darle seguimiento a la estrategia se diseñó e implementó el Observatorio Mexicano de Enfermedades No Transmisibles (OMENT), plataforma en que se divulga información acerca de las enfermedades ECTN, a nivel nacional y estatal y un programa de evaluación denominado "Caminando a la Excelencia" para identificar las áreas de oportunidad para mejorar el desempeño de los diferentes programas de acción. La OMENT es un:

Sistema de Indicadores diseñado para monitorear los avances de la Estrategia Nacional para la Prevención y Control del Sobrepeso, la Obesidad y la Diabetes (ENPCSOD) fue desarrollado por el Instituto Mexicano para la Competitividad A.C. (IMCO) y el Aspen Institute Mexico. El Sistema de Indicadores es una útil herramienta que: permite la comparación transversal de los 32 estados de México en torno a 73 indicadores, para evaluar los factores relacionados con la causalidad de la obesidad, diabetes e hipertensión; permite realizar comparaciones razonables entre factores causales de enfermedades no transmisibles que pueden servir como base para el establecer políticas públicas basadas en evidencia. A pesar de su utilidad, esta herramienta presenta algunas limitaciones: no permite establecer causalidad entre los indicadores de referencia y los indicadores de los tres pilares; no es posible identificar qué componentes ejercen un mayor o menor efecto sobre los indicadores de referencia; no pretende ser la única herramienta que determine el funcionamiento de la ENPCSOD. ${ }^{57}$

56 Estrategia Nacional para la Prevención y el Control del Sobrepeso, la Obesidad y la Diabetes, Secretaría de Salud, México, 2013, pp. 21 y 22.

57 Observatorio Mexicano de Enfermedades No Transmisibles, Universidad Autónoma de Nuevo León, disponible en http://oment.uanl.mx/. 
Por último la estrategia hace énfasis en que las acciones a realizar para la prevención y control del sobrepeso, la obesidad y la diabetes involucran los distintos marcos de acción de la salud, específicamente la Promoción de la salud: acciones para el desarrollo de aptitudes personales para la salud (promoción de alimentación correcta), desarrollo de entornos saludables (escuela y sitios de trabajo), acciones comunitarias (actividades en espacios públicos) e impulso de políticas públicas saludables (acción de vigilancia en alimentos y bebidas, medios masivos de comunicación, orientar al consumidor y establecer medidas fiscales para reducir el consumo de alimentos de alta densidad energética y escaso valor nutrimental). La meta de la estrategia es implementar estrategias que fomenten la conducta de detección temprana en la población, así como también la capacitación del personal en las áreas de prevención porque una detección temprana del padecimiento, aunado a un cambio en los hábitos en alimentación y actividad física, reducen los costos de tratamiento.

\section{EVALUACIONES}

Las evaluaciones realizadas por el Consejo Nacional de la Evaluación de la Política Social de los resultados de los programas para la prevención y combate de la obesidad han encontrado discrepancias entre los objetivos, la operacionalidad, así como también en los cambios en la población beneficiaria.

En el análisis de la documentación disponible encontraron desarticulación entre el nombre, la definición y los indicadores del programa. Además de que, si bien el programa versa sobre la "Prevención contra la obesidad", las acciones puestas en marcha muestran estrategias y líneas de acción enfocadas en la detección, tratamiento y control de sobrepeso, diabetes, dislipidemias y problemas cardiovasculares, pero sus indicadores de resultados sólo estaban enfocados en el control del sobrepeso y excluía a las demás enfermedades crónicas no transmisibles (CONEVAL Informe de Evaluación Específica del Desempeño 2014-2015).

Desde 2015, el Observatorio Mexicano de Enfermedades No Transmisibles (OMENT) publica un Sistema de Indicadores (SI) para monitorear los avances de la Estrategia Nacional para la Prevención y Control del Sobrepeso, la Obesidad y la Diabetes (ENPCSOD). Esta herramienta es un esfuerzo público-privado desarrollado por el Instituto Mexicano para la Competitividad A.C. (IMCO) y el Aspen Institute México. El Sistema de Indicadores fue creado a partir de 67 indicadores, concentrados en 10 componentes, los cuales están agrupados en tres pilares explicativos: Salud pública, Atención médica y Regulación; y un bloque de indicadores de referencia. Los pilares de referencia son (pilar cero): prevalencia de sobrepeso, prevalencia de obesidad, prevalencia de diabetes mellitus tipo 2, prevalencia de hipertensión arterial, mortalidad por diabetes mellitus tipo 2, mortalidad por enfermedades hipertensivas. Los pilares de referencia son los que permiten brindar un seguimiento epidemiológico a la evolución de las estrategias, con base en ellos es posible evaluar el éxito de las estrategias. Con respecto a los 
pilares explicativos están alineados con la ENPCSOD para que útil para el seguimiento de las estrategias por parte de la Secretaría de Salud. ${ }^{58}$

En las páginas siguientes para mejor comprensión del seguimiento y evaluación de los indicadores de la ENPCSOD se presentan unas matrices en las cuales los indicadores de evaluación son referenciados con los pilares, áreas, objetivo general, objetivo específico, meta y componentes de la estrategia. La construcción de las matrices tiene el objetivo de brindar un panorama integral de las dimensiones que entran en juego en la estrategia para conocer la complejidad de la evaluación y evolución del objetivo general de mejorar los niveles de bienestar de la población y contribuir a la sustentabilidad del desarrollo nacional al desacelerar el incremento en la prevalencia de sobrepeso y obesidad en los mexicanos, a fin de revertir la epidemia de las enfermedades no transmisibles, particularmente la diabetes mellitus tipo 2, a través de intervenciones de salud pública, un modelo integral de atención médica y políticas públicas intersectoriales.

Las evaluaciones hechas a las políticas públicas contra la obesidad por parte del Instituto Mexicano de la Competitividad ${ }^{59}$ en el año 2015 para evaluar la calidad a partir de un análisis comparativo (utilizando el marco NOURISHING del Fondo Internacional para la Investigación en Cáncer y tres elementos necesarios para ampliar el panorama de las políticas para complementar el combate a la obesidad: actividad física, lactancia materna y atención médica) arrojó como principales resultados que muchas de las estrategias de política pública puestas en marcha para el combate a la obesidad no cumple con los objetivos propuestos debido a la falta de coordinación entre las jurisdicciones de salud en el país; el cabildeo de las corporaciones y empresas que comercializan alimentos; así como también por la falta de coordinación entre las secretarías (de salud y educación principalmente) para la puesta en marcha y seguimiento de las estrategias destinadas a la comercialización de alimentos en las escuelas.

\section{CONSIDERACIONES FINALES}

En los albores del siglo XXI México tiene dos retos importantes en salud pública, debido a una doble carga de enfermedad: las enfermedades infecciosas asociadas a la pobreza continúan prevaleciendo, y el aumento y dispersión de las enfermedades crónicas no transmisibles sigue en aumento. En relación con el crecimiento de las ECTN la principal meta de las administraciones públicas es la puesta en marcha de estrategias enfocadas en palear el sobrepeso y la obesidad. La obesidad (índice de masa corporal $\geq 30 \mathrm{~kg} / \mathrm{m} 2$ ) es una enfermedad sistémica, crónica y multicausal, que involucra a todos los grupos de edad, de distintas etnias y de todas las clases sociales. Es una enfermedad que ha alcanzado proporciones epidémicas a nivel mundial, razón por la que la Organización Mundial de la Salud (OMS), denomina a la obesidad como la epidemia del siglo.

58 Ídem.

59 El análisis completo de las políticas públicas que forman parte de la Estrategia Nacional de Combate a la Obesidad está en el documento, Instituto Mexicano para la Competitividad, Kilos de más, pesos de menos. Los costos de la obesidad en México, Instituto Mexicano de la Competitividad A. C., México, 2015. 
Las consecuencias de la obesidad son las enfermedades crónicas no transmisibles convertidas en un problema de salud pública a nivel mundial. Contrarrestar la dispersión y aumento de la prevalencia de estas enfermedades requiere de un trabajo multi y transdisciplinario; además de que implica a los tres órdenes de gobierno, la iniciativa privada y la comunidad.

Estudios sobre la dispersión de estas enfermedades encuentran que la raíz estructural, entendida en referencia al orden económico, de las ECTN están directamente ligadas a situaciones de desigualdad a nivel mundial, así como a los ambientes donde se desarrollan las personas. Es por ello, que son un desafío, e implican un reto de gran importancia debido a la magnitud alcanzada, así como la rapidez de su incremento y las consecuencias negativas que afectan directamente la calidad de vida.

En términos económicos el crecimiento de su prevalencia en las regiones del país representa un problema de salud pública que afecta el desarrollo económico y por ello los estudios desde el enfoque médico-epidemiológico es insuficiente debido a su magnitud y el carácter multifactorial que las originan, así como también de sus consecuencias. ${ }^{60}$

La obesidad tiene un origen multifactorial en el que están involucrados factores genéticos, la susceptibilidad, los estilos de vida y las características del entorno; además de que en estos factores influyen diversos determinantes subyacentes, como la globalización, la cultura, la condición económica, la educación, la urbanización y el entorno político y social. Es por ello que un fenómeno tan complejo, en el que tiene un papel predominante tanto el comportamiento individual, como el entorno familiar, comunitario y el ambiente social; la puesta en marcha de políticas públicas, que deben ser integrales, multidimensionales y transversales. Cuyas características dificultan en gran medida darles seguimiento, así como también la interrelación entre los distintos niveles de gobierno y secretarías, ante tal escenario es que los estudios que postulen evaluaciones de las políticas públicas desde la perspectiva etnográfica adquieren pertinencia para proponer medidas acordes con los contextos locales, además de brindarle voz a los enfermos, ya que al fin de las cuentas ellos como actores, son los que viven la enfermedad.

\section{REFERENCIAS.}

AGUILAR VILlanUEVA, Luis, Problemas Públicos y Agenda de Gobierno. Colección Antologías de Política Pública, Miguel Ángel Porrúa, México, 2009.

BELSHAW, Cyril, The Sorcerer's Apprentice: An Anthropology of Public Policy, New York, Pergamon Press, 1976.

BIEHL, Joao y PETRYNA, Adriana, "Critical Global Healht", When People Come First. Critical Studies in Global Health, Estados Unidos, Princeton University Press, 2013, pp. 1-20.

$\overline{60}$ Torres, Felipe y Rojas, Agustín, “Obesidad y Salud Pública en México: Transformación del Patrón Hegemónico de Oferta-Demanda de Alimentos". Revista Problemas del Desarrollo, abril-junio, 2018, 193(49), disponible en https://probdes.iiec.unam.mx. 
BOROFSKY, Robert, “Public Anthropology”, Anthropology News, 40(1), 1999, pp. 6 y 7.

CAMPBELL, Dave, "Anthropology's Contribution to Public Health Policy Development", McGill Journal of Medicine, 2010, 13(1)1, pp. 76-83.

CORDOVA-VILLALOBOS, José Ángel et al. Las enfermedades crónicas no transmisibles en México: sinopsis epidemiológica y prevención integral. Salud pública Méx [online]. 2008, vol.50, n.5 [citado 2019-01-12], pp.419427. Disponible en: $<$ http://www.scielo.org.mx/scielo.php?script=sci_arttext \&pid $=\mathrm{S} 0036-36342008000500015 \& \operatorname{lng}=\mathrm{es} \& \mathrm{nrm}=\mathrm{iso}>$. ISSN 0036-3634.

DAVILA-TORRES, Javier, GONZÁLEZ-IZQUIERDO, José de Jesús y BarreraCruz, Antonio, "Panorama de la obesidad en México". Rev Med Inst Mex Seguro Soc., 53(2), 2015, pp. 240-9.

DIGIACOMO, Susan, "Autobiografía crítica y teoría antropológica. Reflexiones en torno a la enfermedad y la identidad cultural y profesional", en Comelles, Josep, Martorell Poveda, Maria Antonia y Bernal, Mariola, Antropología y enfermería. Campos de encuentro. Un homenaje a Dina Garcés, España, Universitat Rovira i Virgili, 2009.

DUBOIS, Vincent, "Critical policy ethnography", en Fischer, Frank, Torgerson, Douglas, Durnová, Anna y Orsini, Michael (eds.), Handbook of Critical Policy Studies, Estados Unidos, Edward Elgar Publishing, 2017, pp. 462-480.

ELDER, Charles, "El estudio de las políticas públicas; la hechura de las políticas; problemas públicos y agenda de gobierno", Problemas Públicos y Agenda de Gobierno, 3a. ed., edición de Luis F. Aguilar Villanueva, México, Porrúa, 2009 , p. 51.

ELDER, Charles y COBB, Roger, "Formación de la agenda. El caso de la política de los ancianos", Problemas públicos y agenda de gobierno, México, Porrúa, 2009, pp. 77-104.

FRENK, Julio, et al., "La transición Epidemiológica en América Latina". Bol of Sanit Panam, 1991, 111(6); Vera Bolaños, M. (2000) "Revisión crítica a la teoría de la transición epidemiológica”, Papeles de Población, vol. 6, núm. 25, julio-septiembre, 2000.

GUTIÉRREZ, Juan, RIVERA, Juan, SHAMAH, Teresa, OROPEZA, Carlos y HERNÁNDEZ ÁVILA, Mauricio, Encuesta Nacional de Salud y Nutrición 2012, Instituto Nacional de Salud Pública, México, 2012, Disponible en https://ensanut.insp.mx/informes/ENSANUT2012ResultadosNacionales.pdf GUZMÁN-LEÓN, Alicia, "Comparar para construir política pública en tiempos de globalización”, Revista Iberoamericana de Educación Superior, vol. VII, núm. 20, 2016, p. 154.

HEIGHTON, Robert y HEIGHTON, Christy "Applying the Anthropological Perspective to Social Policy", Eddy, Elizabeth y Partridge, William (eds.), Applied Anthropology in America, Estados Unidos, 1987, pp. 390-430.

HERNÁNDEZ MARES, Pablo, "Seis de cada diez mexicanos tienen alguna forma de dislipidemia", Medscape, 2018 Disponible en https://espanol.medscape. com/verarticulo/5903409\#vp_3 consultado el 23 de noviembre de 2018. 
JARAMILLO CARDONA, Martha, Salud y politicas públicas en Baja California: más que un remedio, una enfermedad. Universidad Autónoma de Baja California, México, 2011.

KLEINMAN, Arthur, Patients and healers in the context of culture: An exploration of the borderland between anthropology, medicine, and psychiatry, Estados Unidos, Univ of California Press, 1981.

KLEINMAN, Arthur, The illness narratives: Suffering, healing, and the human condition, Estados Unidos, Universidad de Michigan, 1988.

MACÍAS SANCHEZ, Alejandra y VILLARREAL PÁEZ, Héctor Juan, "Sostenibilidad del gasto público: Cobertura y financiamiento de enfermedades crónicas en México", Ensayos Revista de Economía, 37(1), 2018, pp. 99-134, DOI: http://dx.doi.org/10.29105/ensayos37.1-4.

MENÉNDEZ, Eduardo "La enfermedad y la curación ¿Qué es medicina tradicional?", Alteridades, 1994, 4 (7), 71-83, p. 79.

MÉNDEZ MARTÍNEZ, José Luis, Análisis De Políticas Públicas: Teoría y Casos, México, Colegio De México, 2015.

MICOZZI, Marc "Culture, anthropology, and the return of "complementary medicine"”, Med Anthropol Q, 2002, Dec;16(4), pp. 398-403.

Ministerio de Planificación y Cooperación División Social. Sistema Integrado de Evaluación de Intervenciones Públicas. Documento $\mathrm{N}^{\circ} 4$. Metodología de Análisis de Políticas Públicas: Conceptos y Criterios. Santiago de Chile, diciembre 2000. MOORE, Mark, "Anatomía del problema de la heroína: un ejercicio de definición de problemas", en Aguilar Villanueva, Luis, Problemas Públicos y Agenda de Gobierno, Miguel Ángel Porrúa, México, 2009, pp. 185-214.

MURDOCK, George, "Anthropology and Its Contribution to Public Health", American journal of public health and the nation's health, 1952, 42(1), pp. 7-11.

NELSON, Barbara y LINDENFELD, Thomas, "La formación de una agenda. El caso de maltrato a los niños", Problemas Públicos y Agenda de Gobierno, 3a. ed., edición de Luis F. Aguilar Villanueva, México, Porrúa, 2009, pp. 107 y 108.

OLAVARRÍA GAMBI, Mauricio, Conceptos Básicos en el Análisis de Políticas Públicas. Ecuador, FLACSO, 2007, pp. 11 y 12.

PARKER, Melissa y HARPER, Ian, "The Anthropology of Public Health", Journal of Biosocial Science, 2006, 38(1), 1-5, doi:10.1017/S0021932005001148 RODRÍGUEZ-ABREGO, Gabriela, ESCOBEDO DE LA PEÑA, Jorge, ZURITA G, Beatriz, y RAMÍREZ, Teresita de Jesús, "Esperanza de vida saludable en la población mexicana con seguridad social”, Perinatol Reprod Hum, 2006; 20: 4-18.

ROMAN, Sonia, OJEDA-GRANADOS, Claudia y PANDURO, Arturo, "Genética y evolución de la alimentación de la población en México", Revista de Endocrinología y Nutrición, Vol. 21, No. 1, enero-marzo, 2013, pp. 42-51.

SÁNCHEZ DÍAZ, Natalia, "La carga de la enfermedad", Revista Colombiana de Psiquiatría, XXXIV (2),2005, pp. 298-304. 
SOTO-ESTRADA, Guadalupe, MORENO-ALTAMIRANO, Laura y PAHUA DIAZ, Daniel, "Panorama epidemiológico de México, principales causas de morbilidad y mortalidad", Revista de la Facultad de Medicina de la UNAM, Vol. 59, 6, noviembre-diciembre, 2016, pp. 11-19.

TORRES, Felipe y ROJAS, Agustín, "Obesidad y Salud Pública en México: Transformación del Patrón Hegemónico de Oferta-Demanda de Alimentos". Revista Problemas del Desarrollo, abril-junio, 2018, 193(49), disponible en https://probdes.iiec.unam.mx.

VERA BOLAÑOS, Marta, "Revisión crítica a la teoría de la transición epidemiológica". Papeles de Población, vol. 6, núm. 25, julio-septiembre, 2000, pp. 179-206.

World Health Organization, Global Health Risks. Mortality and burden of disease attributable to selected major risk. WHO Library Cataloguing-in-Publication Data, 2009.

Informes y documentos oficiales

Bases técnicas del Acuerdo Nacional para la Salud Alimentaria. Estrategia contra el sobrepeso y la obesidad (2010). Secretaría de Salud. México.

CONEVAL (2012) Análisis del cumplimiento de los indicadores de resultados, Secretaría de Salud 2007-2012. México.

CONEVAL (2013) Prevención contra la obesidad. Prevención y Control de Sobrepeso, Obesidad y Diabetes. Centro Nacional de Programas Preventivos y Control de Enfermedades, Ficha de Monitoreo. México.

CONEVAL (2014) Prevención contra la obesidad. Prevención y Control de Sobrepeso, Obesidad y Diabetes. Centro Nacional de Programas Preventivos y Control de Enfermedades, Ficha de Monitoreo. México.

CONEVAL (2015) Prevención contra la obesidad. Prevención y Control de Sobrepeso, Obesidad y Diabetes 2014-2015. Valoración de la información de desempeño presentada por el programa. México.

CONEVAL (2017) Prevención contra la obesidad. Prevención y Control de Sobrepeso, Obesidad y Diabetes 2016-2017. Secretaría de Salud, Ficha de Monitoreo. México.

CONEVAL (2018) "Capítulo 2. Diagnósticos de derechos sociales y del ingreso en México, 2.5 Salud”, pp. 104-115. México.

Estrategia Nacional para la Prevención y el Control del Sobrepeso, la Obesidad y la Diabetes (2013). Secretaría de Salud, México.

Global Health Metrics (2018) Global, regional, and national age-sex-specific mortality and life expectancy, 1950-2017: a systematic analysis for the Global Burden of Disease Study 2017. Lancet 2018:392:1684-735.

IMCO (2015) Kilos de más, pesos de menos. Los costos de la obesidad en México. Instituto Mexicano para la Competitividad A.C. México.

Subsecretaría de Integración y Desarrollo del Sector Salud, Dirección General de Evaluación del Desempeño (2015) Informe sobre la salud de los mexicanos, diagnóstico general de la salud poblacional. Secretaria de Salud, México. 
Observatorio Mexicano de Enfermedades No Transmisibles (2018) Sistema de Indicadores para Monitorear los Avances de la Estrategia Nacional para la Prevención y el Control del Sobrepeso, la Obesidad y la Diabetes (ENPCSOD). Componente de Acciones de Salud Pública. Nuevos Indicadores. IMCO, Aspen Institute, Secretaria de Salud, México. Disponible en http:// oment.uanl.mx/indicadores_descargas/doc_metodologico_comp_asp.pdf consultado el 27 de octubre de 2018.

Observatorio Mexicano de Enfermedades No Transmisibles (2018) Actualización de indicadores considerados en el Sistema de Indicadores para monitorear la Estrategia Nacional para la Prevención y el Control del Sobrepesom la Obesidad y la Diabetes (ENPCSOD). Reporte de actualización. IMCO, Aspen Institute, Secretaria de Salud, México. Disponible en http://oment.uanl.mx/ wp-content/uploads/2018/10/reporte_resultados_act_oct18.pdf consultado el 08 de octubre de 2018.

Programa de Acción Específico "Promoción de la Salud y Determinantes Sociales 2013-2018". Programa Sectorial de Salud. México.

Programa Nacional de Salud 2001-2006, Gobierno de la República, Secretaría de Salud. México.

Programa Nacional de Salud 2007-2012, Gobierno de la República, Secretaría de Salud. México.

Plan Nacional de Desarrollo 2013-2018, Gobierno de la República, Programa Sectorial de Salud. México.

Secretaría de Salud, Dirección General de Epidemiología, Observatorio Mexicano de Enfermedades No Transmisibles (2016) Estrategia Nacional para la Prevención y el Control del Sobrepeso, la Obesidad y la Diabetes, Pilar de Salud Pública, Vigilancia Epidemiológica, Panorama Epidemiológico. SSA, DGE, OMENT, México.

Secretaría de Salud, Dirección General de Epidemiología, Observatorio Mexicano de Enfermedades No Transmisibles (2015) Estrategia Nacional para la Prevención y el Control del Sobrepeso, la Obesidad y la Diabetes, Pilar de Salud Pública, Vigilancia Epidemiológica, Panorama Epidemiológico. SSA, DGE, OMENT, México.

Secretaria de Salud (2013) Estrategia Nacional para la Prevención y el Control del Sobrepeso, la Obesidad y la Diabetes. Gobierno de la República, México. Disponible en https://www.gob.mx/cms/uploads/attachment/file/276108/estrategia_sobrepeso_diabetes_obesidad.pdf consultado el 14 de octubre de 2018. Subsecretaría de Prevención y Promoción de la Salud, Dirección General de Promoción de la Salud (2007) Estrategia Nacional de Promoción y Prevención para una Mejor Salud. Secretaría de Salud, Dirección General de Promoción de la Salud, México. 
Norma Oficial Mexicana NOM-008-SSA3-2017, Para el tratamiento integral del sobrepeso y la obesidad. Secretaría de Salud 05/18/2018

Norma Oficial Mexicana, NOM-009-SSA2-2013. Promoción de la salud escolar.

Secretaría de Salud 09/12/2013.

Norma Oficial Mexicana NOM-014-SSA3-2013, Para la asistencia social alimentaria a grupos de riesgo. Secretaría de Salud 11/11/2014.

Norma Oficial Mexicana, NOM-015-SSA2-2010. Para la prevención, tratamiento y control de la diabetes mellitus. Secretaría de Salud 23/11/2010.

Norma Oficial Mexicana, NOM-017-SSA2-2012. Para la vigilancia epidemiológica. Secretaría de Salud. 19/02/2013.

Norma Oficial Mexicana, NOM-028-SCFI-2007. Prácticas Comerciales Elementos de información en las promociones coleccionables y/o promociones por medio de sorteos y concursos. Secretaría de Economía. 05/10/2007.

Modificación a la Norma Oficial Mexicana NOM-030-SSA2-1999, Para la prevención, tratamiento y control de la hipertensión arterial, para quedar como Norma Oficial Mexicana NOM-030-SSA2-2009, Para la prevención, detección, diagnóstico, tratamiento y control de la hipertensión arterial sistémica. Secretaría de Salud. 31/05/2010.

Proyecto de Norma Oficial Mexicana PROY-NOM-030-SSA2-2017, Para la prevención, detección, diagnóstico, tratamiento y control de la hipertensión arterial sistémica. Secretaría de Salud. 19/04/2017.

Norma Oficial Mexicana, NOM-031-SSA2-1999. Para la atención a la salud del niño. Secretaría de Salud. 09/02/2011.

Proyecto de Norma Oficial Mexicana PROY-NOM-031-SSA2-2014, Para la atención a la salud de la infancia. Secretaría de Salud. 25/11/2015.

Norma Oficial Mexicana, NOM-037-SSA2-2012. Para la prevención, tratamiento y control de las dislipidemias. Secretaría de Salud. 13/07/1012.

Norma Oficial Mexicana NOM-040-SSA2-2004. En materia de información en salud. Secretaría de Salud. 30/11/2012.

Norma Oficial Mexicana, NOM-043-SSA2-2012. Servicios básicos de salud. Promoción y educación para la salud en materia alimentaria. Criterios para brindar orientación. Secretaría de Salud. 22/01/2013.

Norma Oficial Mexicana, NOM-051-SCFI/SSA1-2010. Especificaciones generales de etiquetado para alimentos y bebidas no alcohólicas pre envasados - Información comercial y sanitaria. Secretaría de Economía. 05/04/2010.

Modificación a la Norma Oficial Mexicana, NOM-051-SCFI/SSA1-2010. Especificaciones generales de etiquetado para alimentos y bebidas no alcohólicas pre envasados - Información comercial y sanitaria. Secretaría de Salud. $14 / 08 / 2014$.

61 Disponibles en el sitio web de Observatorio Mexicano de Enfermedades No Transmisibles [http://oment.uanl. $\mathrm{mx} /$ normas/]. 
NORMA Oficial Mexicana NOM-131-SSA1-2012, Productos y servicios. Fórmulas para lactantes, de continuación y para necesidades especiales de nutrición. Alimentos y bebidas no alcohólicas para lactantes y niños de corta edad. Disposiciones y especificaciones sanitarias y nutrimentales. Secretaría de Salud. 10/09/2012.

NORMA Oficial Mexicana NOM-251-SSA1-2009, Prácticas de higiene para el proceso de alimentos, bebidas o suplementos alimenticios. Secretaría de Salud. $01 / 03 / 2010$.

Sitios electrónicos

Cámara de Diputados LXIV Legislatura, Centro de Estudios Sociales y de Opinión Pública [http://www5.diputados.gob.mx/index.php/camara/Centros-deEstudio/CESOP].

Sistema de Información en Enfermedades Crónicas [http://www.tablerocronicas. info/DirApp/tableros/OSICEjecIntro.aspx].

Índice de Calidad de la Atención de la Hipertensión en México (ICAHi) [http://201.161.101.68/tablerocronicaspruebas/DirApp/tableros/OSICIntroICAHI.aspx].

Sistema de Indicadores diseñado para monitorear los avances de la Estrategia Nacional para la Prevención y Control del Sobrepeso, la Obesidad y la Diabetes (ENPCSOD) desarrollado por el Instituto Mexicano para la Competitividad A.C. (IMCO) y el Aspen Institute Mexico [http://oment.uanl.mx/ indicadores/].

Elder, Charles, Problemas Públicos y Agenda de Gobierno, 3a. ed., edición de Luis F. Aguilar Villanueva, Porrúa, 2009 